Pacific Journal of Mathematic 


\title{
ON CELLULAR DECOMPOSITIONS OF HILBERT CUBE MANIFOLDS
}

\author{
ZvONKO С̆ERIN
}

\begin{abstract}
M. Brown's notion of a cellular set has proved useful in the study of upper semi-continuous decompositions of finitedimensional manifolds. This paper introduces an infinite-dimensional analogue of it and studies decompositions of Hilbert cube manifolds into cellular sets. Our results give evidence that there is a close analogy between cellular decompositions of finite-dimensional and infinite-dimensional manifolds.
\end{abstract}

1. Introduction. The purpose of this paper is to collect and transliterate into the $Q$-manifold setting a body of the basic "shrinking theorems" and to sort out some of the complications which arise from the infinite-dimensionality. We give several new sufficient conditions on an upper semi-continuous decomposition $G$ of a $Q$-manifold $M$ in order that the quotient space $M / G$ is homeomorphic to $M$.

Let $X$ be a closed subset of a $Q$-manifold $M$. We shall say that $X$ is a cellular subset of $M$ provided $X$ can be represented as $X=$ $\bigcap_{i>0} K_{i}$, where $X \subset \operatorname{int} K_{i}, K_{i} \cong Q, \mathrm{Bd} K_{i} \cong Q$, and $\mathrm{Bd} K_{i}$ is a $Z$-set in $K_{i}$, for each $i>0$.

It is clear that a cellular set $X$ in a $Q$-manifold $M$ has trivial shape and that cellularity depends on the embedding $X \subset M$, not just on $X$ (for example, Wong's wild arc in the Hilbert cube $Q$ [8] is not cellular (see (2.3)(a)). A compact $Z$-set $X$ in $M$ is cellular if and only if $X$ has trivial shape (see $(2.3)(b))$. On the other hand, the cube $Q \times[0,1]$ is cellular in $Q \times[0,2]$ but it is not a $Z$-set in $Q \times[0,2]$.

In $\S 2$ we first prove a comprehensive Theorem (2.4) that gives eleven necessary and sufficient conditions for a proper closed subset $X$ of a compact connected $Q$-manifold $M$ to be cellular. They include the infinite-dimensional version of the famous McMillan's cellularity criterion [32] and the equivalence of cellularity and the property of being pointlike, i.e., that $M-X$ be homeomorphic to $M$ minus a point. We also prove in Theorem (2.8) that a finite-dimensional compactum of trivial shape in the Hilbert cube is cellular if and only if it satisfies cellularity criterion. Hence, finite-dimensional cellular sets in Q-manifolds share many properties with cellular sets in finitedimensional manifolds. The infinite-dimensional cellular sets are more difficult to characterize and recognize which explains numerous complications that arise in attempts to find infinite-dimensional analogues of some results about cellularity in finite-dimensional manifolds.

In the remaining $\S \S 3,4$, and 5 we consider upper semi-continuous 
decompositions $G$ of a $Q$-manifold $M$ into cellular subsets (that we call cellular decompositions) and, equivalently, proper maps $f: M \rightarrow Y$ (called cellular maps) of $M$ onto Hausdorff spaces $Y$ such that for each $y \in Y$ the preimage $f^{-1}(y)$ is a cellular subset of $M$. The separation into three paragraphs corresponds to the three different assumptions that we make about the nature of the set $p\left(H_{G}^{*}\right)$, the projection of the union $H_{G}^{*}$ of all nondegenerate elements $H_{G}$ of $G$ under the quotient map $p: M \rightarrow M / G$. In $\S 3$ we do not make any restrictions on $p\left(H_{G^{*}}^{*}\right)$, in $\S 4$ this set is assumed 0 -dimensional, and in $\S 5$ countable.

Our paper is only an introduction into the study of cellular decompositions of $Q$-manifolds. We leave many questions unanswered and some of them are formulated in the form of three conjectures stated in this introduction. These questions and our results are motivated by corresponding questions and partial results about cellular decompositions of finite-dimensional manifolds.

Let us describe $\S \S 3,4$, and 5 in more detail. The short $\S 3$ contains the important observation (a consequence of the KozlowskiLacher Theorem [25], [28]) that a cell-like map $f: M \rightarrow N$ (i.e., $f$ is a proper map and $f^{-1}(y)$ has trivial shape for each $\left.y \in N\right)$ between $Q$-manifolds is a cellular map. This implies that if we want a decomposition $G$ of a $Q$-manifold $M$ into sets of trivial shape to have the quotient $M / G$ homeomorphic to $M$ then we must assume that $G$ is a cellular decomposition. A modification of Eaton's argument for the existence of dog-bone decompositions for higher dimensional Euclidean spaces (see [12, p. 114]) shows that there are cellular decompositions $G$ of $Q$ with $p\left(H_{G}^{*}\right)$ homeomorphic to a Cantor set and with each nondegenerate element of $G$ a $Z$-set arc such that $Q / G$ is not $Q$. Therefore, in order to get $M / G \cong M$, in addition to assuming that $G$ is cellular, it is necessary to make further assumptions about $G$.

The remainder of $\S 3$ is concerned with the extension to $Q$-manifolds of the appendix of Lacher's paper [27].

In $\S 4$ we study upper semi-continuous decompositions $G$ of a $Q$ manifold $M$ such that $p\left(H_{G}^{*}\right)$ is a 0 -dimensional set in $M / G$ (we call such decompositions 0-dimensional decompositions). Two basic conjectures concerning 0-dimensional decompositions $G$ of a connected $Q$-manifold $M$ are the following.

Conjecture 1. If $G$ is a 0 -dimensional cellular decomposition of a connected $Q$-manifold $M$, then the product $(M / G) \times I$ of $M / G$ with the unit interval $I=[0,1]$ is homeomorphic to $M$.

Conjecture 2. If $G$ is a 0-dimensional decomposition of a (compact) $Q$-manifold $M$ into compact sets such that $M / G \cong M$, then $G$ is 
a cellular decomposition.

The best result supporting Conjecture 1 is Torunczyk's Theorem $5^{\prime}$ in [40] where it was proved that a cell-like decomposition $G$ of a $Q$-manifold $M$ such that $M / G$ is an ANR and $H_{G}^{*}$ is contained in a countable union of finite-dimensional compacta satisfies $(M / G) \times I \cong M$. In the example (4.12) we shall describe a noncellular set $C$ in the Hilbert cube $Q$ which is not a countable union of finite-dimensional compacta such that $(Q / C) \times I \cong Q$.

The suggestion for Conjecture 2 comes from the observations in the $\S 4$. We first recall the notions of weak shrinkable and shrinkable decompositions from [41]. It follows from McAuley's Theorem 2 in $[21, p$. 24] that shrinkability of a decomposition $G$ of a $Q$-manifold $M$ is a sufficient condition for $M / G \cong M$. In the case when $G$ is a cell-like decomposition, this condition is also necessary (see Theorem (4.1)). We prove in Lemma (4.2) that shrinkable decompositions of a Q-manifold are cellular and in Theorem (4.4) that for a 0-dimensional decomposition of a connected $Q$-manifold into compact sets shrinkability and weak shrinkability are equivalent. It follows that a 0-dimensional weakly shrinkable decomposition $G$ of a connected $Q$-manifold $M$ into compact sets is cellular and $M / G \cong M$. Hence, Conjecture 2 is true if a homeomorphism $M / G \cong M$ is a consequence of weak shrinkability of $G$. The conjecture is also true when either $M$ is contractible and $p\left(H_{G}^{*}\right)$ is contained in a 0 -dimensional $Z$-set in $M / G \cong M$ (Theorem (4.5)), or $G$ is a countable decomposition into compact sets of a contractible or a compact connected $Q$-manifold $M$ (Theorem (5.6) and Remark (5.7)). In Conjecture 2 the assumption that $G$ is 0 -dimensional can not be weakened in general because by crossing Bing's example of a decomposition $H$ of the interior of the 3-cell $I^{3}$ into circles and figure eight's such that $I^{3} / H \cong I^{3}([21, \mathrm{p} .7])$ with $Q$ will give us a noncellular decomposition $G$ of $Q$ with $\operatorname{dim} p\left(H_{G}^{*}\right)>0$ such that $Q / G \cong Q$.

We close $\S 4$ with Theorems (4.7), (4.9) and (4.11). The first gives a sufficient condition for a 0-dimensional cellular decomposition $G$ of a $Q$-manifold $M$ to satisfy $M / G \cong M$ analogous to Price's condition in [36]. The second proves that a 0 -dimensional cell-like decomposition $G$ of a $Q$-manifold $M$ equivalent in the sense of ArmentroutLininger-Meyer [2] to a cellular decomposition of $M$ is itself cellular. The last is a restatement of an observation from [34] in the Q-manifold setting used in (4.12) mentioned above.

The final $\S 5$ studies decompositions of $Q$-manifolds with at most countably many nondegenerate elements (such decompositions are called countable decompositions). The best result known for these decompositions is the following special case of Torunczyk's Theorem 3 in [40]. 
Theorem (Torunczyk). If $G$ is a countable decomposition of a $Q$-manifold $M$ into $Z$-sets of trivial shape, then $M / G \cong M$.

The key open question for countable decompositions of $Q$-manifolds, suggested by Bing's countable pointlike decomposition $G$ of $E^{3}$ such that $E^{3} / G$ is not homeomorphic to $E^{3}[21$, p. 12] and a decomposition of the $n$-sphere $(n \geqq 5)$ with analogous properties constructed recently by Daverman [17], is the following.

Conjecture 3. There is a countable cellular decomposition $G$ of the Hilbert cube $Q$ such that $Q / G$ is not homeomorphic to $Q$.

As long as we do not know that the above conjecture is false it is interesting to look for sufficient conditions on a countable cellular decomposition $G$ of a $Q$-manifold $M$ that would imply $M / G \cong M$. We observe (Theorem (5.1)) that this is the case if $H_{G}^{*}$ is a $G_{\delta}$-set in $M$ and in Theorem (5.3) we prove that a decomposition $G$ of $Q$ into a null sequence of tame subcubes of $Q$ (i.e., subsets $K$ of $Q, K \cong Q$, such that $\operatorname{Bd} K \cong Q$ is collared in both $K$ and $Q$-int $K$ ) satisfies $Q / G \cong Q$. Finally, in Theorem (5.6) we show that, for a countable decomposition $G$ of a contractible $Q$-manifold $M$ into compact sets, if the quotient $M / G$ is a $Q$-manifold, then $G$ is cellular and $M / G \cong M$. This result implies (see (5.8)) that the condition from (4.7) is also necessary for a countable decomposition $G$ of a contractible $Q$-manifold $M$ into compact sets to yield a $Q$-manifold as a quotient $M / G$.

We assume that the reader is familiar with notions and results of infinite-dimensional topology. An excellent reference is [12].

I am grateful to Andrea C. F. Shadenhofer for support while I was writing this paper. We also thank the refree for valuable remarks which greatly improved our results and presentation.

\section{Cellular subsets of Q-manifolds.}

Definition 2.1. A closed subset $K$ of a space $M$ is a normal cube in $M$ if $K$ and the boundary $\mathrm{Bd} K$ of $K$ in $M$ are homeomorphic to the Hilbert cube $Q$ and $\mathrm{Bd} K$ is a $Z$-set in $K$.

Observe that $\mathrm{Bd} K$ is a $Z$-set in $K$ if and only if $\mathrm{Bd} K$ has a collar in $K[12$, p. 25]. Hence, normal cubes in $Q$ are the same as closures of open subsets of $Q$ of type $(Q)$ defined by Wong in [43] (see Theorem 2 of that paper).

Definition 2.2. A closed subset $X$ of a $Q$-manifold $M$ is cellular provided $X$ has arbitrarily small open neighborhoods whose closures are normal cubes in $M$. 
EXAMPLE 2.3. (a) Cellular subsets of Q-manifolds have trivial shape since they are intersections of a decreasing sequence of Hilbert cubes $[4$, p. 261]. But cellularity of a closed subset $X$ of a $Q$-manifold $M$ depends on the embedding $X \subset M$ and not just on $X$. For example, it follows from Theorem (2.4) that Wong's wild arc $\alpha$ in the Hilbert cube $Q[8]$ is not cellular because $Q / \alpha$ is not homeomorphic to $Q$.

(b) A $Z$-set $X$ in a compact $Q$-manifold $M$ is cellular if and only if $X$ has trivial shape. Indeed, the first half of this statement follows from (a) above, while the second is a consequence of Theorem (2.4) $(\mathrm{v}) \Rightarrow$ (i) and Theorem 9 in [13] which implies that $M-X$ is homeomorphic to $N-\{p\}$ for some compact $Q$-manifold $N$ and a point $p \in N$.

(c) The standard example of a cellular set that is not a $Z$-set is the half-cube $Q \times[0,1]$ in the product $Q \times[0,2]$. The middle slice $Q \times\{1\}$ in $Q \times[0,2]$ shows that by defining cellular sets as intersections of decreasing sequences of Hilbert cubes (without any conditions on their boundaries) we get an unsatisfactory notion because $(Q \times[0,2]) /(Q \times\{1\})$ is obviously not homeomorphic to $Q \times[0,2]$.

(d) A simple way of constructing cellular sets in $Q$ is through the use of hyperspaces of closed subsets. Let $Y$ be a compact connected ANR and let $A \subset Y$ be a closed connected subset which is the intersection of a decreasing sequence of connected ANR neighborhoods $N_{1} \supset N_{2} \supset N_{3} \supset \cdots$ such that $N_{1} \neq Y$ and $\mathrm{Bd} N_{i}$ is a $Z$-set in $N_{\imath}$ for each $i>0$. Then $X=2^{A}$ is the intersection of a decreasing sequence of Hilbert cubes $\left\{2^{N_{i}}\right\}[15]$. Since the boundary $\mathrm{Bd} 2^{N_{i}}$ of $2^{N_{i}}$ in the Hilbert cube $M=2^{Y}$ is $2^{N_{i}}\left(\mathrm{Bd} N_{i}\right)=\left\{B \in 2^{N_{i}} \mid B \cap \mathrm{Bd} N_{i} \neq \varnothing\right\}$ we see (using Theorem 5.2 in [16]) that $\mathrm{Bd} 2^{N_{i}} \cong Q$ and $\mathrm{Bd} 2^{v_{i}}$ is a $Z$-set in $2^{N_{i}}$. If $A$ has a nonempty interior in $Y$, then $X=2^{A}$ will not be a $Z$-set in $M=2^{Y}$.

The following theorem describes the structure of cellular sets and illustrates the close analogy between that notion and M. Brown's notion of a cellular subset in an $n$-dimensional compact metric space [6]. In its statement we shall use some definitions from [11] that we recall first.

Let $\mathscr{C}$ be a class of topological spaces. A noncompact locally compact space $M$ is $\mathscr{C}$-trivial at $\infty$ provided that for every compact subset $A$ of $M$ there is a compact set $B, B \supset A$, such that every map $f: K \rightarrow M-B$, where $K \in \mathscr{C}$, is null-homotopic in $M-A$. If $\mathscr{P}\left(\mathscr{P}^{k}\right)$ denotes the class of all finite polyhedra (of dimension $\leqq k$ ), then $\mathscr{P}-\left(\mathscr{P}^{k}-\right)$ trivial at $\infty$ spaces are called simply trivial (ktrivial) at $\infty$.

A noncompact locally compact space $M$ is movable at $\infty$ if for every compact set $A$ in $M$ there is a larger compactum $B$ with the 
property that for every compactum $C$ in $M, C \supset A$, and every map $f: K \rightarrow M-B$, where $K \in \mathscr{P}$, there is a homotopy $f_{t}: K \rightarrow M-A$ $(0 \leqq t \leqq 1)$ such that $f_{0}=f$ and $f_{1}(K) \subset M-C$.

THEOREM 2.4. Let $X$ be a proper closed subset of a compact connected Q-manifold $M$. The following statements are equivalent.

( i ) $X$ is cellular in $M$.

( ii ) $X$ is locally shrinkable in $M$ ([21, p. 23]), i.e., for every $\varepsilon>0$ and every open set $U$ containing $X$ there is a homeomorphism $h: M \rightarrow M$ such that $h$ is supported on $U$ and $\operatorname{diam} h(X)<\varepsilon$.

(iii) $X$ is of type $(P)$ in $M$ ([43]), i.e., for each open set $U$ containing $X$ there is a mapping $f$ of $M$ onto itself such that $f$ is supported on $U, 1-1$ outside $X$, and maps $X$ onto a point.

(iv) $M$ and $M / X$ are homeomorphic.

( v ) $X$ has trivial shape and $M / X$ is a Q-manifold.

(vi ) $X$ has trivial shape and the point $\hat{X}$ of $M / X$ corresponding to $X$ is a $Z$-set in $M / X$.

(vii) $X$ is pointlike in the sense that $M-X$ is homeomorphic to $M-\{p\}$ for some point $p \in M$.

(viii) For some point $p \in M$, the Q-manifold $M-\{p\}$ is proper homotopy equivalent to $M-X$.

(ix) $X$ has trivial shape and the Q-manifold $M-X$ has one end and is trivial at $\infty$.

(x) $X$ has trivial shape and the Q-manifold $M-X$ has one end and is movable at $\infty$ and k-trivial at $\infty$ for every $k \geqq 0$.

(xi) $X$ has trivial shape and the Q-manifold $M-X$ has one end and is $\left\{S^{k}\right\}$-trivial at $\infty$ for every $k \geqq 0$ ( $S^{k}$ denotes the $k$-dimensional sphere).

Proof. We shall prove that (i) $\Rightarrow$ (ii), (ii) $\Rightarrow$ (iii), (iii) $\Rightarrow$ (iv), (v) $\Rightarrow$ (iv), (v) $\Leftrightarrow$ (vi), (iv) $\Rightarrow$ (vii), (v) $\Rightarrow$ (ix), (vii) $\Rightarrow$ (i), (vii) $\Rightarrow$ (viii), (viii) $\Rightarrow$ (v), (ix) $\Leftrightarrow(\mathrm{x}),(\mathrm{x}) \Rightarrow(\mathrm{xi})$, and $(\mathrm{xi}) \Rightarrow(\mathrm{v})$.

(i) $\Rightarrow$ (ii). Let $\varepsilon>0$ and an open neighborhood $U$ of $X$ in $M$ be given. Since $X$ is cellular there are normal cubes $K_{1}$ and $K_{2}$ such that $X \subset$ int $K_{2} \subset K_{2} \subset$ int $K_{1} \subset K_{1} \subset U$. It follows from results in [43] and [12] that there is a homeomorphism of quadruples $f:\left(K_{1}, K_{2}\right.$; $\left.\mathrm{Bd} K_{1}, \mathrm{Bd} K_{2}\right) \rightarrow\left(C(Q), C_{1 / 2}(Q) ; Q \times\{1\}, Q \times\{1 / 2\}\right)$ where $C(Q)=(Q \times$ $[0,1]) /(Q \times\{0\})$ is the cone of $Q$ and $C_{s}(Q)=\{[q, t] \in C(Q) \mid 0 \leqq t \leqq s\}$ for every $s \in[0,1]$. By changing only $[0,1]$-coordinates of points in $C(Q)$ we see that for every $\delta>0$ there is a homeomorphism $g_{\delta}: C(Q) \rightarrow$ $C(Q)$ such that $g_{\dot{o}} \mid Q \times\{1\}=\mathrm{id}$ and $\operatorname{diam} g_{\delta}\left(C_{1 / 2}(Q)\right)<\delta$. Let $h: M \rightarrow M$ be an extension by the identity outside $K_{1}$ of a homeomorphism $f^{-1} \circ g_{\dot{o}} \circ f: K_{1} \rightarrow K_{1}$. Clearly, $h$ is supported on $U$ and $\operatorname{diam} h(X)<\varepsilon$ for a sufficiently small $\delta$. 
(v) $\Rightarrow$ (iv). This implication follows from Chapman's CE Approximation Theorem [12].

(vi) $\Rightarrow(v)$. Since $X$ has trivial shape, the quotient $M / X$ is an ANR [23]. It was observed by Ferry [21] (see also [40]) that then $M / X$ is a $Q$-manifold if and only if the point $\hat{X}$ is a $Z$-set in $M / X$.

(viii) $\Rightarrow(\mathrm{v})$. One easily proves that the $Q$-manifold $M-X$ admits a boundary of trivial shape [13]. Hence, $Q$-manifolds $M-X$ and $M-\{p\}$ are homeomorphic near $\infty$ [13, Theorem 7]. That $X$ has trivial shape now follows from the argument below used to prove that (vii) $\Rightarrow$ (i).

(vii) $\Rightarrow$ (i). Let $h: M-\{p\} \rightarrow M-X$ be a fixed homeomorphism and let $U$ be an arbitrary open neighborhood of $X$ in $M$. Let $K \subset$ $h^{-1}(U-X) \cup\{p\}$ be a copy of the Hilbert cube such that $\mathrm{Bd} K \cong Q$ is bicollared in $M$ and let $p \in$ int $K$. We claim that $K^{\prime}=h(K-\{p\}) \cup X$ is a normal cube in $U$ containing $X$ in its interior. Let $L^{\prime}$ denote the union of $M-K^{\prime}$ and a small collar of $\mathrm{Bd} K^{\prime}$ in $K^{\prime}-X$. Then $M=K^{\prime} \cup L^{\prime}$ and $K^{\prime} \cap L^{\prime} \cong \mathrm{Bd} K \times[0,1)$. By Van Kampen's theorem, $\pi_{1}(M)$ is a free product of groups $\pi_{1}\left(K^{\prime}\right)$ and $\pi_{1}\left(L^{\prime}\right)$. But $M-K^{\prime} \cong$ $M-K \cong M-\{p\}$ so that $\pi_{1}\left(M-K^{\prime}\right)=\pi_{1}\left(L^{\prime}\right)=\pi_{1}(M-\{p\})=\pi_{1}(M)$. It follows from Gruško's theorem that $\pi_{1}\left(K^{\prime}\right)$ is trivial. On the other side, the Meyer-Vietoris sequence of the pair $\left(K^{\prime}, L^{\prime}\right)$ gives $H_{q}\left(K^{\prime}\right) \oplus H_{q}\left(L^{\prime}\right)=H_{q}(M)$ for every $q \geqq 2$. We know already that $L^{\prime}$ and $M$ have the same homotopy type. Thus, $H_{q}\left(K^{\prime}\right)=0$ for all $q \geqq 2$. This means that $K^{\prime}$ is a contractible $Q$-manifold and therefore is homeomorphic to the Hilbert cube by [12, Theorem 22.1].

$(\mathrm{x}) \Rightarrow$ (ix). We observed in $[9, \mathrm{p} .139]$ that a movable at $\infty$ space that is also $k$-trivial at $\infty$, for every nonnegative integer $k$, is trivial at $\infty$.

$(\mathrm{xi}) \Rightarrow(\mathrm{v})$. We shall show that the $Q$-manifold $M-X$ admits a boundary of trivial shape [13]. This will imply by [13, Theorem 9] that $M / X \cong(M-X) \cup \infty$ is a $Q$-manifold. Clearly, it suffices to prove that for every open neighborhood $U$ of $X$, the inclusion $U-X \hookrightarrow U$ is a homotopy equivalence. In order to do this, by Whitehead's theorem, we must check that the pair $(U, U-X)$ is $n$-connected for every $n \geqq 0$, i.e., that every map $f:\left(\Delta^{n}, \mathrm{Bd} \Delta^{n}\right) \rightarrow$ $(U, U-X)$ of the standard $n$-simplex $\Delta^{n}$ is homotopic (rel $\mathrm{Bd} \Delta^{n}$ ) to a map into $U-X$.

Pick neighborhoods $V_{0} \subset V_{1} \subset \cdots \subset V_{n-1} \subset V_{n}=U$ of $X$ such that inclusions $V_{i} \hookrightarrow V_{i+1}$ are null-homotopic in $V_{i+1}$ and every map $\psi: S^{k} \rightarrow$ $V_{i}-X$ is null-homotopic in $V_{i+1}-X$, for every $0 \leqq k \leqq n$ and $i=$ $0,1, \cdots, n-1$.

Consider an arbitrary map $f:\left(\Delta^{n}, \mathrm{Bd} \Delta^{n}\right) \rightarrow(U, U-X)$. Cover $f^{-1}(X)$ with the interiors of a finite number of disjoint $n$-cells $\tau_{1}, \cdots, \tau_{m}$ which do not meet $\mathrm{Bd} \Delta^{n}$ such that the $\tau_{i}$ are simplices of 
a subdivision $T$ of $\Delta^{n}$ and are such that $f\left(\tau_{i}\right) \subset V_{0}$. Let $M^{k}=\bigcup_{i=1}^{m}(k-$ skelton of $\left.\tau_{i}\right), 0 \leqq k \leqq n$, and let $N=\Delta^{n}-\bigcup_{i=1}^{m}\left(\right.$ int $\left.\tau_{i}\right)$.

We shall prove, by induction, that for every $s, 0 \leqq s \leqq n$, there is a homotopy $\varphi_{t}^{s}: M^{s} \rightarrow V_{s}$ such that $\varphi_{0}^{s}=f \mid M^{s}, \varphi_{1}^{s}\left(M^{s}\right) \subset V_{s-1}-X$ and $\varphi_{t}^{s}\left|M^{s} \cap N=f\right| M^{s} \cap N$.

Indeed, assume $\varphi_{t}^{s-1}$ has already been defined. If $\sigma \in M^{s}$ is an arbitrary $s$-simplex, then $\varphi_{1}^{s-1} \mid \mathrm{Bd} \sigma: \mathrm{Bd} \sigma \rightarrow V_{s-2}-X$ extends to a map $\psi: \sigma \rightarrow V_{s-1}-X$. Since the inclusion $V_{s-1} \hookrightarrow V_{s}$ is null-homotopic, the map on $(\mathrm{Bd} \sigma) \times I \cup \sigma \times\{0,1\}$ defined as $\psi$ on $\sigma \times\{1\}$, as $f \mid \sigma$ on $\sigma \times\{0\}$ and as $\varphi_{t}^{s-1} \mid \mathrm{Bd} \sigma$ on $(\mathrm{Bd} \sigma) \times I$ can be extended to a homotopy in $V_{s}$ of $f \mid \sigma$ with the map $\psi$. Piecing all such homotopies together will give us a required homotopy $\varphi_{t}^{s}$.

We now extend $\varphi_{t}^{n}$ to all of $\Delta^{n}$ by defining $\varphi_{t}^{n}|N=f| N$ for each $t$. Thus $f$ is homotopic to a map $\varphi_{1}^{n}: \Delta^{n} \rightarrow U-X$ rel $N$ and hence rel Bd $\Delta^{n}$.

The remaining implications in the above plan of our proof are obvious.

REMARK 2.5. The implication (ix) $\Rightarrow$ (i) can be regarded as the infinite-dimensional version of McMillan's cellularity criterion [32] while the implication (vii) $\Rightarrow$ (i) is the extension to $Q$-manifolds of a theorem due to Christenson and Osborne [14] for $n$-manifolds. Simple examples show that in general our assumptions can not be weakened. In particular, the requirement that $M$ be compact and connected is essential in the implication (vii) $\Rightarrow(i)$. Indeed, let $Y=\left(S^{1} \times Z^{+} \vee\right.$ $Z) \cup \infty$ be the one-point compactification of the disjoint union of the product of the circle $S^{1}$ and nonnegative integers $Z^{+}$and the integers $Z$. Let $\varphi: Y \rightarrow Q$ be an embedding of $Y$ onto a $Z$-set in $Q$. Let $M=Q-\varphi\left(\left(S^{1} \times N \vee Z\right) \cup \infty\right)$ and $X=\varphi\left(S^{1} \times\{0\}\right)$. Then $X$ is pointlike in $M$ but is not cellular.

REMARK 2.6. For a simply-connected compact $Q$-manifold $M$ in (v), (vi), (ix), and (x) (but not in (xi) as is clear from the discussion below) we can weaken the requirement that $X$ has trivial shape with the condition that $X$ has trivial reduced Čech cohomology with integer coefficients. Indeed, in (3.4) we shall show this for (v) while in the above proof that (vi) implies (v) this time we need an improvement of [20] due to Kozlowski (see [13, p. 207]) in order to get that $M / X$ is an ANR without using the fact that $X$ has trivial shape.

Finally, for (ix) and (x) we first observe that because $M-X$ is one-ended and $\left\{S^{1}\right\}$-trivial at $\infty$ there are arbitrarily large compact $Q$-manifolds $M_{0}$ in $M-X$ such that $\mathrm{Bd} M_{0}$ is simply-connected and collared both in $M_{0}$ and in $M$ - int $M_{0}$ (see [13, p. 198]). Since $M$ is simply-connected it follows from Van Kampen theorem that 
$M$ - int $M_{0}$ is simply-connected and therefore that $X$ is an $U V^{1}$ compactum. Applying Hurewicz theorem [28] (see also [31]) we get that $X$ is an $U V^{k}$-compactum, for each $k \geqq 0$. It is well-known (see [4], [19]) that, in general, we can not conclude now that $X$ has trivial shape (or, equivalently, that it is an $U V^{\infty}$-compactum). This will follow however in the cases when $X$ is either movable or it has finite (fundamental) dimension (see [19] and [35]). In our situation we proceed to this conclusion as follows.

The idea again is to show that $M-X$ admits a boundary of trivial shape. Since $X$ is the intersection of open neighborhoods $U$ of finite homotopy type and since the above proof of the implication $(\mathrm{xi}) \Rightarrow(\mathrm{v})$ can be applied to get that the inclusion $U-X \hookrightarrow U$ is a homotopy equivalence, $M-X$ is tame at $\infty$ [13]. By the Practical Boundary Theorem in [13] we see that $M-X$ admits a boundary. This boundary must have trivial shape because $M-X$ is trivial at $\infty$ (see [11, Theorem (3.2)]).

The following amusing application of (2.4) improves Theorem 2 in [43].

Corollary 2.7. Let $X$ be a compactum of trivial shape in the Hilbert cube $Q$ and let $W$ be a component of $Q-X$. If $W$ is $\left\{S^{k}\right\}$ trivial at $\infty$ for every $k \geqq 0$, then $W$ is homeomorphic to $Q \times[0,1)$ (and $W^{*}=W \cup X$ is homeomorphic to $Q$ if $X$ is a $Z$-set in $W^{*}$ ).

Proof. Let $Z$ denote the union of other components of $Q-X$. Then $Z^{*}=Z \cup X$ and $W^{*}$ are compact subsets of $Q$ such that $Z^{*} \cup W^{*}=Q$ and $Z^{*} \cap W^{*}=X$. By [4, p. 322], $Z^{*}$ has trivial shape. Since $Q-Z^{*}=W$, we can apply Theorem (2.4) (xi) $\Rightarrow$ (vii) to show that $Z^{*}$ is pointlike in $Q$ and hence get the result because $Q$ $\{$ point $\} \cong C(Q)-\{$ vertex $\} \cong Q \times[0,1)[12]$. The statement within parentheses follows from [20].

We turn now our attention to the characterization of cellularity for finite-dimensional sets. Surprisingly, McMillan's Theorem 1 in [32] holds for them without any additional assumptions. Observe that a compact set $X$ in the interior of a manifold $M^{n}$ satisfies cellularity criterion ([28, p. 539]) if and only if $M-X$ is $\left\{S^{1}\right\}$-trivial at $\infty$.

Theorem 2.8. Let $X$ be a finite-dimensional compactum of trivial shape in the Hilbert cube $Q$. Then $X$ is cellular in $Q$ if and only if $M=Q-X$ is $\left\{S^{1}\right\}$-trivial at $\infty$.

Proof. We established in (2.4) that $\left\{S^{1}\right\}$-triviality at $\infty$ of $M$ is a necessary condition for $X$ to be cellular. We shall now prove its 
sufficiency when $X$ is finite-dimensional.

Since $X$ is finite-dimensional, $M$ has one end and therefore is $L C^{1}$ at $\infty\left[13\right.$, p. 198]. Also, $H_{*}(M)$ is trivial [26]. By the corollary to the Practical Boundary Theorem in [13], we know that there is a compact $Q$-manifold $N$ and a $Z$-set $B$ in $N$ such that $N-B \cong M$. Since we can embedd a cone $C(B)$ on $B$ into $N$ in such a way that $C(B)$ is a $Z$-set in $N$ and the base of $C(B)$ agree with $B$, applying Anderson-Chapman Homeomorphism Extension Theorem [12, p. 30] we see that there are maps of $N$ onto $N / B \cong Q / X$ with arbitrarily small preimages. It follows by Eilenberg's theorem [3, p. 221] that $N$ is homotopy dominated by the absolute retract $Q / X[23]$. Hence, $N$ is contractible and therefore $N \cong Q[12, \mathrm{p} .36]$. This is a key fact which implies that $B$ has trivial shape. Indeed, by Proposition (6.5) in [10], $B$ has trivial reduced Čech cohomology. But we also know that $B$ is approximatively 1-connected (or, equivalently, an $U V^{1}$-compactum) [11, Theorem (3.2)]. Applying Hurewicz theorem in the shape theory (see [4], [28], and [31]), we get that $B$ has all homotopy pro-groups trivial. In other words, $B$ is approximatively $k$-connected for every $k \geqq 0$ (or, equivalently, an $U V^{k}$-compactum for every $k \geqq 0$ ). This implies (by [11, Theorem (3.2)]) that $M$ is $\left\{S^{k}\right\}$-trivial at $\infty$ for every $k \geqq 0$. Now, from $(2.4)(x i) \Rightarrow(i)$ we conclude at last that $X$ is cellular.

REMARK 2.9. In the above theorem it suffices to assume that $X$ has trivial reduced Čech cohomology with integer coefficients because we can apply an argument from $\S 3$ in [29] to get that $X$ is an $U V^{1}$-compactum which implies that $X$ has trivial shape (see (2.6)).

EXAMPLE 2.10. In the first version of this paper the author erroneously claimed that a finite-dimensional subset of a compact connected $Q$-manifold with $\left\{S^{1}\right\}$-trivial at $\infty$ complement is a $Z$-set. In particular, that finite-dimensional cellular sets in $Q$ are $Z$-sets. Now we shall sketch the construction of a cellular arc in $Q$ which is not a $Z$-set in $Q$.

The first step is to observe that there is a wild $\operatorname{arc} \alpha$ in $Q$ with simply-connected noncontractible complement. The arc $\alpha$ can be constructed by adapting methods in [18] to the Hilbert cube using techniques from [44]. Note that $(Q / \alpha) \times[0,1] \cong Q$. Hence, the cone $C(Q / \alpha)=(Q / \alpha) \times[0,1] /(Q / \alpha) \times\{1\}$ is also homeomorphic with $Q$ [12] (because $(Q / \alpha) \times\{1\}$ is a $Z$-set of trivial shape in $(Q / \alpha) \times[0,1])$. The required arc $\beta$ is the image of $\{\hat{\alpha}\} \times[0,1]$ in $C(Q / \alpha)$ under the quotient map ( $\hat{\alpha}$ denotes the point of $Q / \alpha$ corresponding to $\alpha$ ).

Indeed, the complement of $\beta$ in $C(Q / \alpha)$ is homeomorphic to $(Q-\alpha) \times[0,1)$. This implies that $\beta$ is not a $Z$-set in $C(Q / \alpha)$ because 
its complement is not contractible and that $\beta$ is cellular (by (2.8)) because $(Q-\alpha) \times[0,1)$ is $\left\{S^{1}\right\}$-trivial at $\infty$ (see Lemma (3.2) in [8] and Theorem 7 in [32]).

REMARK 2.11. With obvious modifications the proofs in $\S 4$ of [32] and Theorem (2.8) give us the following results.

(i) Let $X$ be a finite-dimensional compactum in the Hilbert cube $Q$. If $Q-X$ is locally 1-connected at each point of $X$ [32], then every compactum of trivial shape which is a subset of $X$ is cellular in $Q$.

(ii) Let $A$ be a cellular arc in the Hilbert cube. Then each subarc of $A$ is cellular.

REMARK 2.12. In several proofs in this section we referred to results from [13]. The paper [13] is based on Siebenmann's unpublished thesis [38] and on [39] and is nonelementary because of a complicated algebra involved. Since we used [13] only in the "trivial case" where there is no algebra (when the arguments in [13] are much simpler) and because of the elementary nature of the rest of our paper we shall briefly describe how to avoid [13].

(a) Example (2.3) (b): The cellularity of $Z$-sets of trivial shape is established in [12], where the Engulfing argument on pp. 14-15 works in general, employing Theorem 19.4 on pp. 30-31.

(b) Theorem (2.4) (viii) $\Rightarrow(\mathrm{v})$ : This is the same as (a) in spirit; using the fact that $M-X$ is proper-homotopy equivalent to $M-\{p\}$, we provide by controlled $Z$-set engulfing a homeomorphism of $U \times[0,1) \cup N \times[0,1]$ onto $U \times[0,1]$, where $U$ is a closed neighborhood of $\infty$ in $M-X$ and $N$ is a compact neighborhood in $U$ of the boundary of $U$ in $M$. Now by the same $Z$-set engulfing argument Chapman gives in Chapter $\mathrm{V}$ of [12], we show that some neighborhood $V \subset U$ of $\infty$ in $M-X$ is homeomorphic with $Q \times[0,1)$, which is a neighborhood of $\infty$ in $M-\{p\}$.

(c) Theorem (2.4) (xi) $\Rightarrow(\mathrm{v})$ : Our proof has essentially established that $M-X$ is trivial at $\infty$, i.e., $(\mathrm{xi}) \Rightarrow(\mathrm{ix})$; now we show that (ix) $\Rightarrow$ (vii) by rigidifying to a proper homotopy equivalence, which is easy in this case.

(d) Remark (2.6) and Theorem (2.8): The corollary to the Practical Boundary Theorem can be proved elementary following the proof in [5] (this is an unpublished result of Chapman).

3. Cellular decompositions and cellular maps. An upper semicontinuous decomposition $G$ of a $Q$-manifold $M$ is a cellular decomposition provided that each element of $G$ is cellular in $M$. Similarly, a proper map $f: M \rightarrow Y$ of a $Q$-manifold $M$ onto a Hausdorff space 
$Y$ is called a cellular map if the preimage $f^{-1}(y)$ of every point $y \in Y$ is cellular in $M$.

An important class of cellular maps is that of cell-like maps between Q-manifolds. This follows from the following more general observation.

THEOREM 3.1. Let $f: M \rightarrow N$ be a cell-like map of Q-manifolds. $A$ compact subset $Y$ of $N$ is cellular in $N$ if and only if $X=f^{-1}(Y)$ is cellular in $M$.

Proof. We recall that a cell-like map between ANRs is a hereditary shape equivalence and a proper homotopy equivalence at the same time [25] (see also [27]). This implies that $X$ has trivial shape if and only if $Y$ has trivial shape and that $M-X$ is trivial at the end of $M-X$ corresponding to $X$ if and only if $N-Y$ is trivial at the end of $N-Y$ corresponding to $Y$. Now, we apply the implication (ix) $\Rightarrow$ (i) in (2.4).

CoROLlaRy 3.2. A proper map $f: M \rightarrow N$ of Q-manifolds is cellular if and only if it is cell-like.

REMARK 3.3. It is clear from the proof of (3.1) that the above corollary can be improved by assuming that $N$ is just a locally compact ANR each point $y$ of which has an open neighborhood $V$ such that $V-\{y\}$ is trivial at the end of $V-\{y\}$ corresponding to $y$.

We shall now extend the appendix of Lacher's paper [27] to $Q$ manifolds. These results show that certain types of isolated singularities do not exist.

Let $M$ be a $Q$-manifold, $Y$ a Hausdorff space, and let $f: M \rightarrow Y$ be a proper map onto $Y$. Define two "singular sets" as follows:

$$
C_{f}=\left\{y \in Y \mid f^{-1}(y) \text { is not cellular in } M\right\},
$$

and

$$
Q_{f}=\{y \in Y \mid Y \text { is not locally } Q \text { at the point } y\} .
$$

Recall [27] that an onto map $f: M \rightarrow N$ between $Q$-manifolds is strongly acyclic if and only if the reduced Čech cohomology of $f^{-1}(y)$ is trivial for each $y \in N$.

TheOREM 3.4. Let $M$ and $N$ be Q-manifolds and let $f: M \rightarrow N$ be a proper, strongly acyclic map of $M$ onto $N$. If $M$ is simplyconnected, then $C_{f}$ has no isolated points.

Proof. Suppose that a point $y_{0} \in N$ has an open neighborhood $V$ 
such that $f^{-1}(y)$ is cellular in $M$ for each $y \in V, y \neq y_{0}$. Let $U=$ $f^{-1}(V), \quad V_{0}=V-\left\{y_{0}\right\}$, and $U_{0}=U-f^{-1}\left(y_{0}\right)$. Observe that $f \mid U_{0}$ : $U_{0} \rightarrow V_{0}$ is a cellular map of $Q$-manifolds. Hence, $f \mid U_{0}$ is proper homotopic to a homeomorphism $h: U_{0} \rightarrow V_{0}\left[12\right.$, p. 105]. But $y_{0}$ has arbitrarily small compact neighborhoods $W$ such that $\left(W-\left\{y_{0}\right\}, \mathrm{Bd} W\right) \cong$ $(Q \times[0,1), Q \times\{0\})$. Hence, $f^{-1}\left(y_{0}\right)$ is the intersection of $Q$-manifold neighborhoods $Z=h^{-1}\left(W-\left\{y_{0}\right\}\right) \cup f^{-1}\left(y_{0}\right)$ such that $\mathrm{Bd} Z=Q$. By using the Van-Kampen theorem and the assumption that $M$ is simplyconnected we see that $Z$ is simply-connected so that $f^{-1}\left(y_{0}\right)$ has property $U V^{1}$. It follows that $f^{-1}\left(y_{0}\right)$ is an $U V^{k}$-compactum for all $k \geqq 0$ (see [27]). But $f^{-1}\left(y_{0}\right)$ is also movable because neighborhoods $Z$ can be compressed by a homotopy as close to $f^{-1}\left(y_{0}\right)$ as we please. This implies that $f^{-1}\left(y_{0}\right)$ has trivial shape [19] and therefore that it is cellular in $M$.

EXAMPLE 3.5. The condition that $M$ is simply-connected can not be dropped. Indeed, let $X$ be an acyclic ANR such that $\pi_{1} X$ is not trivial ( $X$ can be obtained from a nonsimply connected homology $n$-sphere, $n \geqq 3$, by deleting the interior of a simplex). The product $X \times Q$ is a nonsimply connected $Q$-manifold [12, Theorem 44.1]. Let $N$ be a normal cube in $X \times Q$. Then a map $f: X \times Q \rightarrow N / B d N$, obtained by shrinking $X \times Q$-int $N$ to a point, is a strongly acyclic map of $Q$-manifolds such that $C_{f}$ is a single point (see [27]).

THEOREM 3.6. Let $M$ be a compact Q-manifold and let $Y$ be a Hausdorff space. If $f: M \rightarrow Y$ is a cellular map, then $Q_{f}$ has no isolated points.

Proof. We shall present two proofs. The first is more involved because it uses Chapman's CE Approximation Theorem.

Let $y_{0} \in Y$ and suppose that $y_{0}$ has an open neighborhood $V$ such that $V-\left\{y_{0}\right\}$ is a $Q$-manifold. Let $W=f^{-1}\left(V-\left\{y_{0}\right\}\right)$. The restriction $f \mid W: W \rightarrow V-\left\{y_{0}\right\}$ is a cell-like map of $Q$-manifolds. By [12, Corollary 43.2], $f \mid W$ is proper homotopic to a homeomorphism $W \rightarrow$ $V-\left\{y_{0}\right\}$. Hence, if $\varepsilon$ is the end of $W$ corresponding to $f^{-1}\left(y_{0}\right)$, we see from $(2.4)(\mathrm{i}) \Rightarrow(\mathrm{v})$ that $\left[W \cup f^{-1}\left(y_{0}\right)\right] / f^{-1}\left(y_{0}\right) \cong W \cup \varepsilon \cong V$ is a $Q$-manifold.

We can arrive to this conclusion also in the following way. By Kozlowski and Lacher ([25] and [28], resp.), $f \mid W$ is a proper homotopy equivalence. It follows from (2.4) (i) $\Rightarrow$ (ix) that $V-\left\{y_{0}\right\}$ is trivial at the end corresponding to $y_{0}$. Now invoke [37, Theorem (4.2)] or [7, Theorem (4.2)] to conclude that $V$ is an ANR and $\left\{y_{0}\right\}$ is a $Z$-set in $V$. Applying Ferry's observation [20] we see that $V$ is a Q-manifold. 
4. O-dimensional cellular decompositions. Let $G$ be an upper semi-continuous decomposition of a $Q$-manifold $M$. Let $H_{G}$ be a collection of all nondegenerate elements of $G$ and let $H_{G}^{*}$ denote the union of all elements in $H_{G}$. If the natural projection $p=p_{G}: M \rightarrow$ $M / G$ of $M$ onto the quotient space $M / G$ of $G$ carries $H_{G}^{*}$ onto a 0 -dimensional set we call $G$ a 0 -dimensional decomposition. In this section we shall mostly consider 0-dimensional cellular decompositions.

We first recall two definitions on shrinkability of decompositions from [41]. A decomposition $G$ of $M$ is called weakly shrinkable if for each open set $U$ containing $H_{G}^{*}$ and each $\varepsilon>0$, there is a homeomorphism $h: M \rightarrow M$ supported on $U$ such that $\operatorname{diam} h(g)<\varepsilon$ for each $g \in H_{G}$. It is named shrinkable in case that for each covering $\mathscr{C}$ of $H_{G}^{*}$ by saturated open subsets of $M$ (i.e., by open sets $U \subset M$ satisfying $p^{-1} p(U)=U$ ), for each $\varepsilon>0$, and for an arbitrary homeomorphism $h: M \rightarrow M$ there exists a homeomorphism $f: M \rightarrow M$ such that

(1) $f$ agrees with $h$ outside $\mathscr{C}^{*}=\mathrm{U}\{U \mid U \in \mathscr{Q}\}$, and

(2) for each $g \in G$, (a) $\operatorname{diam} f(g)<\varepsilon$ and (b) there exists $D \in \mathscr{C}$ such that $h(D) \supset h(g) \cup f(g)$.

THEOREM 4.1. Let $G$ be a cell-like decomposition of a Q-manifold $M$. Then $M / G \cong M$ if and only if $G$ is a shrinkable decomposition.

Proof. If $G$ is shrinkable, then McAuley's Theorem 2 in [21, p. 24] implies $M / G \cong M$.

Conversely, suppose $M / G \cong M$. Then the map $p: M \rightarrow M / G=N$ is a cell-like map of $Q$-manifolds. By Corollary 43.2 in [12] such a map is a near-homeomorphism, i.e., (see 26.1 in [12]) for every open cover $\mathscr{C}$ of $M$ and every open cover $\mathscr{V}$ of $N$ there is a homeomorphism $f=f_{i, y}: M \rightarrow M$ such that $p \circ f$ is $\mathscr{Y}$-close to $p$ and for each $g \in G$ there is $U \in \mathscr{Q}$ with $f(g) \subset U$.

Now, let $\mathscr{Q}$ be a covering of $H_{G}^{*}$ by saturated open sets of $M$ and let $\varepsilon>0$ be given. By an argument on page 31 of [41] it suffices to find a shrinking homeomorphism $f$ satisfying (1) and (2) above in the case when $h=\mathrm{id}$, the identity. In order to do this, let $\mathscr{U}^{\prime}$ be a normal cover [22] of the open set $\mathscr{C}^{*}$ refining $\mathscr{C}$ and consisting of open sets with a diameter $<\varepsilon$. Let $\mathscr{y}$ be an open covering of the open set $p\left(\mathscr{U}^{*}\right)$ such that the star St $\mathscr{Y}$ of $\mathscr{V}$ refines $p(\mathscr{C})=$ $\{p(U) \mid U \in \mathscr{U}\}$. For a cell-like map $p \mid \mathscr{U}^{*}: \mathscr{U}^{*} \rightarrow p\left(\mathscr{U}^{*}\right)$ of $Q$-manifolds construct a homeomorphism $f^{\prime}=f_{\mathscr{U}^{\prime}, \mathscr{V}}: \mathscr{Q}^{*} \rightarrow \mathscr{U}^{*}$. Clearly, $f^{\prime}$ can be extended by the identity outside $\mathscr{C}^{*}$ to a required homeomorphism $f: M \rightarrow M$.

By replacing $n$-cells with normal cubes in the proof of Lemma 2.4 in [41] we get the proof of the following. 
LEMmA 4.2. Let $G$ be a shrinkable decomposition of a Q-manifold $M$ into compact sets. Then $G$ is cellular.

The best information about 0 -dimensional weakly shrinkable decompositions of $Q$-manifolds available is the following special case of Lamoreaux's general theorems in [30] (in particular, consult Theorems 4,10 , and 13 in [30]).

THEOREM 4.3. Let $G$ be a weakly shrinkable 0-dimensional decomposition of a connected $Q$-manifold $M$ into compact sets. Then $M / G \cong M$ and each element $g$ of $G$ is pointlike.

In the case when $M$ is compact, combining (4.3), (4.2), and (2.4) (vii) $\Rightarrow$ (i) it follows that a weakly shrinkable 0-dimensional decomposition $G$ of a compact connected $Q$-manifold $M$ is cellular and $M / G \cong M$. With only minor changes in the proof of Lemma 2.5 in [41] (Voxman's Lemma 2.1 was established for an arbitrary locally compact connected metric space in [30, Theorem 3] while his Lemma 2.3 follows from our Theorem (4.3) and the fact that a pointlike compact subset of a connected $Q$-manifold must be connected) we can prove the following result which implies that in the above argument the requirment that $M$ is compact can be removed.

THEOREM 4.4. Suppose $G$ is a 0-dimensional decomposition of a connected Q-manifold $M$ into compact sets. Then $G$ is weakly shrinkable if and only if $G$ is shrinkable.

THEOREM 4.5. Let $G$ be a 0-dimensional decomposition of a contractible Q-manifold $M$ into compact sets such that $M / G$ is a $Q$-manifold and $p\left(H_{G}^{*}\right)$ is contained in a 0 -dimensional $Z$-set in $M / G$. Then $G$ is a cellular decomposition.

Proof. Let $x \in p\left(H_{G}^{*}\right)$ and let $K$ be a normal cube neighborhood of $x$ in $M / G$. We can identify $K$ with the cone $C(Q)$ of $Q$ (see the proof of (i) $\Rightarrow$ (ii) in (2.4)). The assumption about $p\left(H_{G}^{*}\right)$, the fact that every compact 0 -dimensional space embedds into the Cantor set, homogeneity of the Cantor set, and Anderson's Homeomorphism Extension Theorem [12, Theorem 11.1] imply that there is a homeomorphism $h: C(Q) \rightarrow C(Q)$ such that $h\left(p\left(H_{G}^{*}\right) \cap K\right)$ is contained in the standard Cantor set in the interval $I_{0}=\{[\mathcal{O}, t] \in C(Q) \mid 0 \leqq t \leqq 1\}$ (where $\mathscr{O} \in Q$ is a fixed point) and $h(x)$ is the vertex $[\mathscr{O}, 0] \in I_{0}$. It is clear that $h(x)$ has arbitrarily small normal cube neighborhoods $N$ whose boundaries are bicollared in $C(Q)-h\left(p\left(H_{G}^{*}\right) \cap K\right)$. This gives that $M$ can be written as the union $M=p^{-1} h^{-1}(N) \cup\left(M-p^{-1} h^{-1}(\right.$ int $\left.N)\right)$ 
with the intersection $p^{-1} h^{-1}(N) \cap\left(M-p^{-1} h^{-1}(\operatorname{int} N)\right)=p^{-1} h^{-1}(\operatorname{Bd} N)$ homeomorphic to $Q$. It follows from [22, p. 49] and [12, p. 36] that $p^{-1} h^{-1}(N)$ is a contractible $Q$-manifold and thus is a normal cube neighborhood of $p^{-1}(x)$ in $M$. Hence, $G$ is a cellular decomposition.

REMARK 4.6. The above theorem is not true for noncontractible $Q$-manifolds $M$ because if $f: Q \rightarrow M$ is an embedding of $Q$ onto a $Z$-set in $M$, then the mapping cylinder $M(f)$ of $f$ is homeomorphic to $M$ and $M(f) / M$ is homeomorphic to $Q$ [12]. It is not true even if we assume that $M / G \cong M$. Indeed, for each negative (nonnegative) integer $n$ attach to the product $Q \times R$ of $Q$ with the real line $R$ a copy $M_{n}$ of $M(f) / M(M(f))$ along the base $Q \times\{0\}$ onto $Q \times\{n\}$ and let $N$ be the new $Q$-manifold. By shrinking the copy of $M$ in $M_{0} \subset N$ to a point we shall obviously get $N$ again. This example shows that Conjecture 2 is not true in general without the assumption that $M$ is compact.

Another (besides (4.3)) sufficient condition for a cellular decomposition $G$ of a connected $Q$-manifold $M$ to give $M$ as a decomposition space $M / G$ is the following analogue of Price's main result in [36]. In (5.8) below we shall show that for countable decompositions of contractible $Q$-manifolds into compact sets this condition is also necessary.

THEOREM 4.7. Let $G$ be an upper semi-continuous decomposition of a connected Q-manifold $M$. Suppose that for each element $g \in H_{G}$ there exists a sequence $\left\{K_{i}^{g}\right\}_{i=1}^{\infty}$ of normal cubes in $M$ such that for each positive integer $i$

(1) $K_{i+1}^{g} \subset \operatorname{int} K_{i}^{g}$,

(2) $\bigcap_{i=1}^{\infty} K_{i}^{g}=g$, and

(3) $\left(\mathrm{Bd} K_{i}^{g}\right) \cap H_{G}^{*}=\varnothing$.

Then the decomposition space $M / G$ is homeomorphic to $M$.

Proof. Since the decomposition $G$ is 0-dimensional (every point of $p\left(H_{G}^{*}\right)$ has arbitrarily small open neighborhoods in $p\left(H_{G}^{*}\right)$ with empty boundaries) and cell-like, by combining Theorems (4.1) and (4.4) and Theorem 10 in [30], we see that it suffices to prove the theorem for the case $M=Q \times[0, \infty)$.

The proof for this case can be given following Price's proof of Theorem 1.4 in [36] provided we can prove an analogue of his Lemma 1.1 (stated below as Lemma (4.8)) and then replace the balls $B(k)$ in the construction on page 431 of [36] with Hilbert cubes $Q \times[0, k]$.

LEMMA 4.8. Let $G$ be a monotone decomposion of the cone $C(Q)=$ $(Q \times[0,1]) /(Q \times\{0\})$ on $Q$ and assume that no nondegenerate element 
of $G$ intersects the top $Q_{1}=Q \times\{1\}$ of $C(Q)$. Then given $\varepsilon>0$, there exists a homeomorphism $f$ of $C(Q)$ onto itself such that $\operatorname{diam} f(g)<\varepsilon$ for every $g \in G$, and $(2) f$ is pointwise fixed on $Q_{1}$.

Proof. The homeomorphism $f$ affects only the second coordinate of every point $[q, t] \in C(Q)$ and it pushes all elements of diameter $\geqq \varepsilon$ into the $(\varepsilon / 2)$-neighborhood of the vertex $v=[q, 0]$, it does not stretch much those elements whose diameters are $<\varepsilon$, and it keeps $Q_{1}$ pointwise fixed. The details of the construction of $f$ are clear if one is familiar with the proof of Lemma 1.1 in [36].

The following definition is adopted from [2]. Let $F$ and $G$ be decompositions of a $Q$-manifold $M$. Then $F$ and $G$ are equivalent provided there is a homeomorphism $h: M / F \rightarrow M / G$ such that $h$ maps the closure $\mathrm{Cl}\left(p_{F}\left(H_{F}^{*}\right)\right)$ onto $\mathrm{Cl}\left(p_{G}\left(H_{G}^{*}\right)\right)$.

THEOREM 4.9. Suppose $F$ and $G$ are equivalent cell-like decompositions of a compact connected Q-manifold $M$ such that the closures $\mathrm{Cl}\left(p_{F}\left(H_{F}^{*}\right)\right)$ and $\mathrm{Cl}\left(p_{G}\left(H_{G}^{*}\right)\right)$ are both finite-dimensional. If $F$ is cellular, then $G$ is also cellular.

Proof. For $g \in G$ select $f \in F^{\prime}$ such that $h\left(p_{F}(f)\right)=p_{G}(g)$, where $h: M / F \rightarrow M / G$ is a homeomorphism with the property that $h\left(\mathrm{Cl}\left(p_{F}\left(H_{F^{*}}^{*}\right)\right)\right)=\mathrm{Cl}\left(p_{t i}\left(H_{* i}^{*}\right)\right)$. Consider the diagram

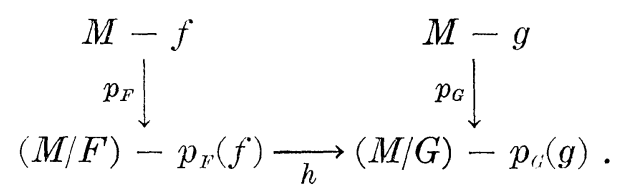

Since $M / F$ and $M / G$ are ANR's (by a theorem of Kozlowski [25]; this is also a consequence of an unpublished result of R. D. Anderson proved in [42]), maps $p_{F}$ and $p_{G}$ are proper homotopy equivalences [25], [28]. This implies that $M-g$ is one-ended and trivial at $\infty$ because $M-f$ is one-ended and trivial at $\infty$ (since $f$ is cellular). It follows from (2.4) (ix) $\Rightarrow$ (i) that $g$ is cellular.

Now we recall a simple condition from [34] which implies that shrinking compact sets $C$ and $A$ in a $Q$-manifold $M$ produces equivalent decompositions.

Let $N_{\varepsilon}(X)$ denote the open $\varepsilon$-neighborhood of a subset $X$ in a metric space.

DeFinition 4.10. Let $C$ be a compact subset of a $Q$-manifold $M$ and let $A$ be a closed subset of $C$. We shall say that $C$ compresses towards $A$ if for every $\varepsilon>0$ there is a homeomorphism $f: M \rightarrow M$ fixing $M-N_{\varepsilon}(C)$ and $A$ such that $f(C) \subset N_{\varepsilon}(A)$. 
The results in [34] can be stated in our situation as follows.

THEOREM 4.11. Let $C$ and $A, A \subset C$, be compact subsets of a Q-manifold $M$. If $C$ compresses towards $A$, then there is a homeomorphism $h: M / C \rightarrow M / A$ with $h(\hat{C})=\hat{A}$.

We close this section with an example of a strongly infinitedimensional noncellular set $C$ in the Hilbert cube $Q$ and a wild $\operatorname{arc} A$, $A \subset C$, in $Q$ such that $C$ compresses towards $A$. It follows from Theorem (4.11) and [8] that $(Q / C) \times I \cong(Q / A) \times I \cong Q$. Hence, the decomposition $G$ of $Q$ with the only nondegenerate element $C$ is an example of a noncellular decomposition of $Q$ such that $(Q / G) \times I \cong Q$ and to which we can not apply Torunczyk's theorem mentiond in the introduction.

EXAMPLE 4.12. Let $Q_{0}$ be a compact convex infinite-dimensional subset of the Hilbert space $l_{2}$ and assume that $Q_{0}$ contains the origin 0 of $l_{2}$. Let $Q$ denote the suspension of $Q_{0}$ geometrically realized as the join of $Q_{0} \times\{0\}$ with points $(0,-1)$ and $(0,1)$ in the product $l_{2} \times R$ of $l_{2}$ with the real line. Let $A$ be a wild arc in $Q \cap\left(Q_{0} \times\right.$ $(-\infty, 0])$ [8] which intersects $Q_{0} \times\{0\}$ only at the point $(0,0)$. Next, we select a compact convex infinite-dimensional set $Q_{1 / 2}$ in $Q \cap\left(l_{2} \times\right.$ $\{1 / 2\})$ such that $Q_{1 / 2}$ does not intersect the boundary of $Q$ in $l_{2} R$. Let $C^{\prime}$ denote the join of $Q_{1 / 2}$ with $(0,0)$ and put $C=C^{\prime} \cup A$. By [24], $Q_{0}, Q, Q_{1 / 2}$, and $C^{\prime}$ are Hilbert cubes. Using linear transformations along rays from the point $(0,0)$ and phasing them out outside the $\varepsilon$-neighborhood of $C$ it is easy to see that $C$ compresses toward $A$.

5. Countable cellular decompositions. In this final section we shall consider countable decompositions $G$ of a connected $Q$-manifold $M$, i.e., decompositions with at most countably many nondegenerate elements.

Since each cellular set in a $Q$-manifold $M$ is locally shrinkable in $M$ (see (2.4) (i) $\Rightarrow$ (ii)) from McAuley's Theorem 1 in $[21$, p. 23] we immediately get.

THEOREM 5.1. Suppose that $G$ is a countable cellular decomposition of a Q-manifold $M$ such that $H_{G}^{*}$ is a $G_{\delta}$ in $M$. Then the decomposition space $M / G$ is homeomorphic to $M$.

Our next theorem gives another sufficient condition for a countable decomposition $G$ of the Hilbert cube $Q$ in order that $Q / G \cong Q$. The restriction is on the nature of elements of $G$ (they are tame cubes) and on their size ( $G$ is a null family, i.e., for every $\varepsilon>0$ 
only finitely many elements of $G$ have diameter $>\varepsilon$ ). The proof of this theorem follows closely Meyer's proof in [33] of a similar result for $E^{3}$.

Definition 5.2. A Hilbert cube $K$ in $Q$ is called a tame cube provided $\mathrm{Bd} K \cong Q$ and $\mathrm{Bd} K$ is bicollared in $Q$.

THEOREM 5.3. Let $G$ be an upper semi-continuous decomposition of $Q$ such that $G$ is a null family and each nondegenerate element of $G$ is a tame cube in $Q$. Then the decomposition space $Q / G$ is homeomorphic to $Q$.

Proof. It is clear that $G$ is a countable cell-like decomposition so that by (4.1) and (4.4) it suffices to prove that $G$ is weakly shrinkable. This will be accomplished in Lemma (5.5) below whose proof is based on the following technical result.

We shall consider the cone $C(Q)$ as obtained from the Hilbert cube

$$
Q=\left\{\left(x_{i}\right) \in l_{2}\left|x_{1}=1,\right| x_{i} \mid \leqq \sqrt{3} \cdot 2^{-i} \text { for } i>1\right\}
$$

by joining each point in $Q$ by a straight line segment with the origin $0=(0,0, \cdots)$. For each $\eta, 0<\eta<1$, let

$$
C_{\eta_{1}}(Q)=\left\{\left(x_{i}\right) \in C(Q) \mid x_{1} \leqq \eta\right\} .
$$

LEMmA 5.4. Let $\delta>0$ and $0<\varepsilon<1 / 2$ be real numbers, and let $\left\{K_{i}\right\}_{i>0}$ be a null family of mutually disjoint compact sets of diameter $<\delta$ in $C(Q)-C_{1 / 2}(Q)$. Then there exists a homeomorphism $h$ of the cone $C(Q)$ onto itself such that $\operatorname{diam} h\left(C_{1 / 2}(Q)\right)<\delta, \operatorname{diam} h\left(K_{i}\right)<\delta$ for every $i>0$, and $h \mid C(Q)-C_{(1 / 2)+\varepsilon}(Q)=\mathrm{id}$.

Proof. Since $C_{\eta}(Q)$ has diameter $(\sqrt{5} / 2) \eta$, if $\delta>\sqrt{5} / 4$, the identity function has the desired properties. Therefore, assume that $\delta \leqq \sqrt{5} / 4$. Let $s$ be $(2 \delta) /(2+\sqrt{5})$. There is a positive integer $M$ such that $0<(\sqrt{5} / 2)((1 / 2)-M s)<\delta$.

Suppose that $\delta<a<b<c<(1 / 2)+\varepsilon$. Let $f_{a, b, c}$ be a homeomorphism of the closed unit interval $[0,1]$ onto itself such that

(i) if $x \in[0, a], f_{a, b, c}(x)=x-(x / a) s$,

(ii) if $x \in[a, b], f_{a, b, c}(x)=x-s$,

(iii) if $x \in[b, c], f_{a, b, c}(x)=x-((c-x) /(c-b)) s$, and

(iv) if $x \in[c, 1], f_{a, b, c}(x)=x$.

Let $G_{a, b, c}$ be a homeomorphism of $C(Q)$ onto itself defined by $G_{a, b, c}(x q)=$ $f_{a, b, c}(x) q$ for $q \in Q$ and $0 \leqq x \leqq 1$. Observe that $d\left(G_{a, b, c}(x q), x q\right) \leqq$ $(\sqrt{5} / 4)\left|f_{a, b, c}(x)-x\right|$. Hence, $d(x q, y r)<s$ in $C(Q)$ implies $d\left(G_{a, b, c}(x q)\right.$, $\left.G_{a, b, c}(y r)\right) \leqq \delta$ 
It is now clear how to proceed as in the proof of Lemma 1 in [33] to finish the proof.

Lemma 5.5. Suppose $G$ is an upper semi-continuous decomposition of $Q$ into points and tame cubes in $Q$ such that $G$ is null family. Then $G$ is weakly shrinkable.

Proof. Let $\alpha>0$ and an open set $U$ containing $H_{G}^{*}$ be given. Let $H_{G}=\left\{g_{1}, g_{2}, \cdots\right\}$ be the enumeration of nondegenerate elements of $G$. Since $H_{G}$ is a null family, there is an index $N$ such that $i>N$ implies diam $g_{i}<\alpha$. Let $V_{1}, V_{2}, \cdots, V_{N}$ be mutually disjoint open sets such that if $i \leqq N$, then $g_{i} \subset V_{i} \subset U$.

For each index $1 \leqq i \leqq N$, a homeomorphism $h_{i}$ of $Q$ onto itself will be constructed such that $h_{i}$ will be supported on $V_{i}, h_{i}$ will shrink $g_{i}$ to a set of diameter $<\alpha$, and $h_{i}$ will stretch no element of $H_{G}$ to a diameter as much as $\alpha$. The composition $f=h_{N} \circ \cdots \circ h_{1}$ is the required shrinking homeomorphism.

Let $i$ be a positive integer less than or equal to $N$. Since $g_{i}$ is a tame cube in $Q$, by results in [43], there is a homeomorphism $k: Q \rightarrow C(Q)$ such that $k\left(g_{i}\right)=C_{1 / 2}(Q)$ and $k\left(\mathrm{Bd} g_{i}\right)=\mathrm{Bd} C_{1 / 2}(Q)$. There exists $\gamma>0$ such that $\left.C_{(1 / 2)+\gamma}(Q)\right) \subset k\left(V_{i}\right)$. Let $K=k^{-1}\left(C_{(1 / 2)+r}(Q)\right)$. Observe that $k^{-1}$ is uniformly continuous on $C_{(1 / 2)+\gamma}(Q)$ and $k$ is uniformly continuous on $K$. Thus there exists $\delta>0$ such that if $L \subset C_{(1 / 2)+r}(Q)$ and $\operatorname{diam} L<\delta$ then $\operatorname{diam} k^{-1}(L)<\alpha$; and there exists $\beta>0$ such that if $M \subset K$ and $\operatorname{diam} M<\beta$ then $\operatorname{diam} k(M)<\delta$. Also, there exists $\varepsilon, 0<\varepsilon<\gamma$, such that if $j>N$ and $g_{j} \cap k^{-1}\left(C_{(1 / 2)+\varepsilon}(Q)\right) \neq \varnothing$, then $\operatorname{diam} g_{j}<\beta$ and $g_{j} \subset K$.

Let $D=\left\{d_{1}, d_{2}, \cdots\right\}$ be the enumeration of the set $\left\{k\left(g_{j}\right) \mid g_{j} \cap\right.$ $k^{-1}\left(C_{(1 / 2)+\varepsilon}(Q)\right) \neq \varnothing$ and $\left.j>N\right\}$. Since $k$ is uniformly continuous on $K$, $D$ is a null family. By Lemma (5.4), there exists a homeomorphism $G: C(Q) \rightarrow C(Q)$ such that $\operatorname{diam} G\left(C_{1 / 2}(Q)\right)<\delta, \operatorname{diam} G\left(d_{j}\right)<\delta$ for every $d_{j} \in D$, and $G \mid C(Q)-C_{(1 / 2)+\varepsilon}(Q)=$ id. Let $h_{i}=k^{-1} \circ G \circ k$.

The verification that $f$ is a required homeomorphism is the same as the argument on page 603 in [33].

We return now to Conjecture 2 and prove that it is true for countable decompositions of both contractible and compact connected $Q$-manifolds. This result resembles Armentrout's main theorem in [1] for the 3-sphere.

THEOREM 5.6. Let $G$ be a countable decomposition of a contractible Q-manifold $M$ into compact sets such that $M / G$ is a Q-manifold. Then $G$ is a cellular decomposition and $M / G \cong M$.

Proof. By Corollary (3.2) and Chapman's CE Approximation 
Theorem, it suffices to prove that $G$ is a cell-like decomposition. Let $g \in G$ and let $K$ be a normal cube in $M / G$ which contains $p(g)$ in its interior. Let $h: K \rightarrow C(Q)$ be a homeomorphism which takes the point $p(g)$ to the vertex $v$ of the cone $C(Q)$. We can assume that the projections of points $\{h \circ p(\widetilde{g}) \mid \widetilde{g} \in G$ and $p(\widetilde{g}) \in K\}$ onto the $[0,1]$-factor of $C(Q)$ are rational. Hence, we see that $p(g)$ is the intersection of Hilbert cubes $\left\{K_{i}\right\}_{i>0}$, selected from Hilbert cubes $\left\{h^{-1}\left(C_{r}(Q)\right) \mid 0<\eta<1\right.$ is irrational\}, such that $\mathrm{Bd} K_{i} \cong Q$ and $\left(\mathrm{Bd} K_{i}\right) \cap p\left(H_{G}^{*}\right)=\varnothing$. Then $L=p^{-1}\left(K_{i}\right)$ has a boundary homeomorphic to the boundary of $K_{i}$. Hence, $M=L \cup \mathrm{Cl}(M-L)$ where $L \cap \mathrm{Cl}(M-L)=\mathrm{Bd} L \cong \mathrm{Bd} K_{i} \cong \mathrm{Q}$. It follows that $L$ is an $\mathrm{AR}[22, \mathrm{p}$. 49] and we conclude that $g=$ $p^{-1} p(g)$ is cell-like.

REMARK 5.7. The argument for the implication (viii) $\Rightarrow$ (i) in Theorem (2.4) essentially shows that the contractibility hypothesis of Theorem (5.6) is superfluous if $M$ is compact because $g$ is pointlike (since $\quad M \cong \mathrm{Cl}\left(M-p^{-1} h^{-1}\left(C_{\tau_{1}}(Q)\right)\right) \cup p^{-1} h^{-1}\left(C_{\tau_{1}}(Q)-\operatorname{int} C_{\tau_{2}}(Q)\right) \cup \cdots \cup$ $p^{-1} h^{-1}\left(C_{\tau_{n}}(Q)\right)$ for each finite decreasing sequence $\tau_{1}, \tau_{2}, \cdots, \tau_{n}$ of irrational numbers and we have almost all of the $p^{-1} h^{-1}\left(C_{\tau_{i}}(Q)-\operatorname{int} C_{\tau_{i+1}}(Q)\right)$ contractible).

Corollary 5.8. Let $G$ be a countable decomposition of a contractible Q-manifold $M$ into compact sets such that $M / G$ is a Q-manifold. Then for each element $g \in H_{G}$ there exists a sequence $\left\{K_{i}^{o}\right\}_{i>0}$ of normal cubes in $M$ such that for each positive integer $i$ conditions (1)-(3) in the statement of Theorem (4.7) hold.

Proof. We know from Theorem (5.6) that $G$ is a cellular decomposition and $M / G \cong M$. Let $g \in H_{G}$ and let the natural projection $p: M \rightarrow M / G$ maps $g$ into the point $x$ of $M / G$. Since $M / G$ is a $Q$ manifold, there is a normal cube $K$ in $M / G$ such that $x \in \operatorname{int} K$. Let $h: K \rightarrow C(Q)$ be a homeomorphism which takes $x$ into the vertex $v$ of $C(Q)$, and let $r: C(Q) \rightarrow[0,1]$ be a projection of $C(Q)$ onto its $[0,1]$ factor (it maps a point $[q, t] \in C(Q)=(Q \times[0,1]) /(Q \times\{0\})$ into $t$ ). By assumption, $r \circ h\left(K \cap p\left(H_{G}^{*}\right)\right)$ is a countable subset of $I=[0,1]$. We can assume that this set consists entirely of rational numbers and that $r \circ h(x)=0$.

Let $t, 0<t<1$, be an irrational number. The restriction $p \mid(r \circ h \circ p)^{-1}([0, t)):(r \circ h \circ p)^{-1}([0, t)) \rightarrow(r \circ h)^{-1}([0, t))$ is a cell-like map of $Q$-manifolds. By applying CE Approximation Theorem [12, Corollary 43.2] with a normal cover relative to $(r \circ h \circ p)^{-1}(t)$ we find a homeomorphism of $(r \circ h \circ p)^{-1}([0, t])$ onto $(r \circ h)^{-1}([0, t])$ which agrees with $p$ on the set $(r \circ h \circ p)^{-1}(t)$. Hence, $(r \circ h \circ p)^{-1}([0, t])$ is a normal cube in $M$ containing $g$ in its interior whose boundary $(r \circ h \circ p)^{-1}(t)$ 
does not intersect $H_{G}^{*}$. By taking $t$ sufficiently close to 0 this neighborhood will be as close to $g$ as we please.

\section{REFERENCES}

1. S. Armentrout, Upper semi-continuous decompositions of $E^{3}$ with at most countably many non-degenerate elements, Ann. of Math., 78 (1963), 605-618.

2. S. Armentrout, L. L. Lininger and D. V. Meyer, Equivalent decompositions of $R^{3}$, Pacific J. Math., 24 (1968), 205-227.

3. K. Borsuk, Theory of retracts, Monografie Matematyczne 44, Warsaw, 1969.

4. - Theory of shape, Monografie Matematyczne 59, Warsaw, 1975.

5. W. Browder, J. Levine and G. R. Livesay, Finding a boundary for an open manifold, Amer. J. Math., 87 (1965), 1017-1028.

6. M. Brown, A proof of the generalized Schoenflies theorem, Bull. Amer. Math. Soc., 66 (1960), 74-76.

7. Z. Čerin, $n$-docility at infinity and compactifications of $L C^{n}$ spaces, Glasnik Mat., 12 (1977), 161-174.

8. ㄴ, Hilbert cube modulo an arc, Fund. Math., 101 (1978), 19-27.

9. _- Homotopy at in finity of proper maps, Glasnik Mat., 13 (1978), 135-154.

10. - $\mathscr{C}_{p}$-movable at in finity spaces, compact ANR divisors and property $U V W^{n}$, Publ. Inst. Math., 23 (1978), 53-65.

11. - Homotopy properties of locally compact spaces at in finity-triviality and movability, Glasnik Mat., 13 (1978), 347-370.

12. T. A. Chapman, Lectures on Hilbert cube manifolds, Regional conference series in mathematics no. 28, Amer. Math. Soc., 1976.

13. T. A. Chapman and L. C. Siebenmann, Finding a boundary for a Hilbert cube manifold, Acta Math., 137 (1976), 171-208.

14. C. O. Christenson and R. P. Osborne, Pointlike subsets of a manifold, Pacific J. Math., 24 (1968), 431-435.

15. D. W. Curtis and R. M. Schori, $2^{X}$ and $C(X)$ are homeomorphic to the Hilbert cube, Bull. Amer. Math. Soc., 80 (1974), 927-931.

16. - Hyperspaces which characterize simple homotopy type, General Top. and Appl., 6 (1976), 153-165.

17. R. J. Daverman, A non-shrinkable decomposition of $S^{n}$ determined by a null sequence of cellular sets, Proc. Amer. Math. Soc., 75 (1979), 171-176.

18. D. G. DeGryse and R. P. Osborne, $A$ wild Cantor set in $E^{n}$ with simply connected complement, Fund. Math., 86 (1974), 9-27.

19. J. Dydak, The Whitehead and Smale theorems in shape theory, Dissertationes Mathematicae, 156 (1978) 1-55.

20. S. Ferry, Completions of Q-manifolds which are Q-manifolds, (preprint).

21. M. F. Fort, Jr. (editor), Topology of 3-Manifolds, Prentice-Hall, 1962.

22. S. T. Hu, Theory of Retracts, Wayne State University Press, Detroit, 1965.

23. D. M. Hyman, ANR divisors and absolute neighborhood contractibility, Fund. Math., 62 (1968), 61-73.

24. O. H. Keller, Die Homeomorphie der kompakten Mangen in Hilbertschen Raum, Math. Ann., 105 (1931), 748-758.

25. G. Kozlowski, Images of ANRs, (preprint).

26. N. S. Kroonenberg, Characterization of finite-dimensional Z-sets, Proc. Amer. Math. Soc., 43 (1974), 421-427.

27. R. C. Lacher, Cell-like mappings II, Pacific J. Math., 35 (1970), 649-660.

28. _ Cell-like mappings and their generalizations, Bull. Amer. Math. Soc., 83 (1977), 495-552.

29. — A cellularity criterion based on codimension, Glasnik Mat., 11 (1976), 135-140. 
30. J. W. Lamoreaux, Decomposition of metric spaces with a 0-dimensional set of non. degenerate elements, Canad. J. Math., 21 (1969), 202-216.

31. S. Mardešić and Š. Ungar, The relative Hurewicz theorem in shape theory, Glasnik Mat., 9 (1974), 317-327.

32. D. R. McMillan, Jr., A criterion for cellularity in a manifold, Ann. of Math., 79 (1964), 327-337.

33. D. V. Meyer, A decomposition of $E^{3}$ into points and a null family of tame 3-cells is $E^{3}$, Ann. of Math., 78 (1963), 600-604.

34. — $E^{3}$ modulo a 3-cell, Pacific J. Math., 13 (1963), 193-196.

35. S. Nowak, On the fundamental dimension of approximatively 1-connected compacta, Fund. Math., 89 (1975), 61-79.

36. T. M. Price, A necessary condition that a cellular upper semi-continuous decomposition of $E^{n}$ yield $E^{n}$, Trans. Amer. Math. Soc., 122 (1966), 427-435.

37. R. B. Sher, Docility at in finity and compactifications of ANR's, Trans. Amer. Math. Soc., 221 (1976), 213-224.

38. L. C. Siebenmann, The obstruction to finding a boundary for an open manifold of dimension greater than five, Doctoral dissertation, Princeton Univ., 1965.

39. _ Infinite simple homotopy types, Indag. Math., 32 (1970), 479-495.

40. H. Torunczyk, On CE-images of the Hilbert cube and characterization of Q-manifolds, (prtprint).

41. W. L. Voxman, On the shrinkability of decompositions of 3-manifolds, Trans. Amer. Math. Soc., 150 (1970), 27-39.

42. J. E. West, Mapping Hilbert cube manifold to ANRs, Ann. of Math., 106 (1977), 43. R. Y. T. Wong, Extending homeomorphisms by means of collarings, Proc. Amer. Math. Soc., 19 (1968), 1443-1447.

44 .

A wild Cantor set in the Hilber cube, Pacific. J. Math., 24 (1968), 189-193.

Received December 14, 1978 and in revised form September 6, 1979.

UNIVERSITY OF ZAGREB

41001 ZagReB, P.P. 187

YUGOSLAVIA 



\section{PACIFIC JOURNAL OF MATHEMATICS}

\section{EDITORS}

DoNALD BABBITT (Managing Editor)

University of Galifornia

Los Angeles, California 90024

HUgo RossI

University of Utah

Salt Lake City, UT 84112

C. C. MOORE AND ANDREW OGG

University of California

Berkeley, CA 94720
J. DugunduI

Department of Mathematics

University of Southern California

Los Angeles, California 90007

R. Finn and J. Milgram

Stanford University

Stanford, California 94305

\section{ASSOCIATE EDITORS}
E. F. BeCKenbaCh
B. H. NeumanN
F. WOLF
K. YoSHIDA

\section{SUPPORTING INSTITUTIONS}

UNIVERSITY OF BRITISH COLUMBIA

CALIFORNIA INSTITUTE OF TECHNOLOGY

UNIVERSITY OF CALIFORNIA

MONTANA STATE UNIVERSITY

UNIVERSITY OF NEVADA, RENO

NEW MEXICO STATE UNIVERSITY

OREGON STATE UNIVERSITY

UNIVERSITY OF OREGON
UNIVERSITY OF SOUTHERN CALIFONIA STANFORD UNIVERSITY UNIVERSITY OF HAWAII UNIVERSITY OF TOKYO UNIVERSITY OF UTAH WASHINGTON STATE UNIVERSITY UNIVERSITY OF WASHINGTON 


\section{Pacific Journal of Mathematics}

\section{Vol. 91, No. $1 \quad$ November, 1980}

Harvey Leslie Abbott, Extremal problems on nonaveraging and nondividing

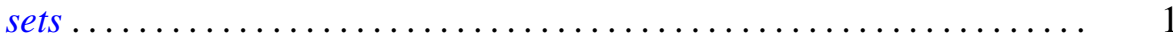

Marine Bruce Abrahamse and Stephen D. Fisher, Mapping intervals to

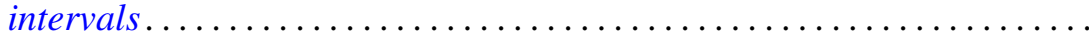

William Wells Adams, The best two-dimensional Diophantine

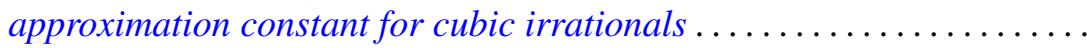

Marilyn Breen, A quantitative version of Krasnosel'skiu 's theorem in

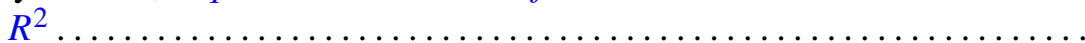

Stephen LaVern Campbell, Linear operators for which $T^{*} T$ and $T T^{*}$

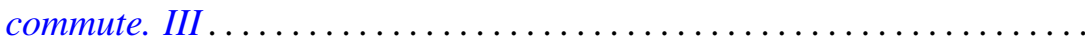

Zvonko Cerin, On cellular decompositions of Hilbert cube manifolds ......

J. R. Choike, Ignacy I. Kotlarski and V. M. Smith, On a characterization

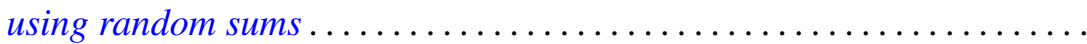

Karl-Theodor Eisele, Direct factorizations of measures .............. 79

Douglas Harris, Every space is a path component space ............. 95

John P. Holmes and Arthur Argyle Sagle, Analytic H-spaces, Campbell-Hausdorff formula, and alternative algebras.............

Richard Howard Hudson and Kenneth S. Williams, Some new residuacity criteria ..........................................

V. Karunakaran and Michael Robert Ziegler, The radius of starlikeness for a class of regular functions defined by an integral ....

Ka-Sing Lau, On the Banach spaces of functions with bounded upper

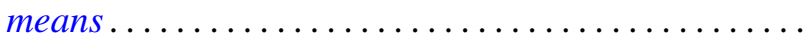

Daniel Paul Maki, On determining regular behavior from the recurrence formula for orthogonal polynomials................

Stephen Joseph McAdam, Asymptotic prime divisors and going down...

Douglas Edward Miller, Borel selectors for separated quotients ..

Kent Morrison, The scheme of finite-dimensional representations of an algebra

Donald P. Story, A characterization of the local Radon-Nikodým property by tensor products

Arne Stray, Two applications of the Schur-Nevanlinna algorithm ...

N. B. Tinberg, The Levi decomposition of a split $(B, N)$-pair ...

Charles Irvin Vinsonhaler and William Jennings Wickless, A theorem on quasi-pure-projective torsion free abelian groups of finite rank... 